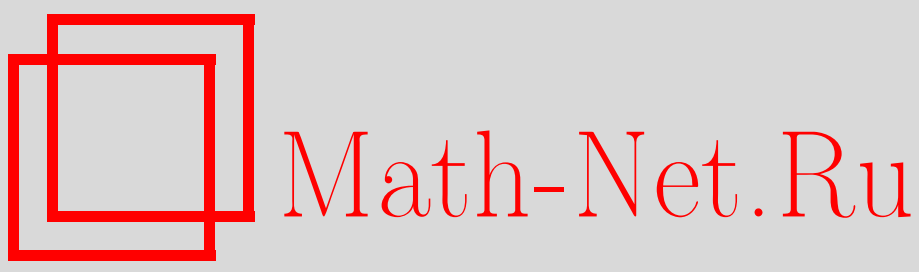

А. А. Толстоногов, Существование оптимального управления без предположения выпуклости в эволюционной системе первого порядка, Матем. сб., 2001, том 192, номер 9, 125-142

DOI: https://doi.org/10.4213/sm597

Использование Общероссийского математического портала Math-Net.Ru подразумевает, что вы прочитали и согласны с пользовательским соглашением

http://www . mathnet.ru/rus/agreement

Параметры загрузки:

IP : 54.237 .206 .68

26 апреля 2023 г., 16:18:10 


\author{
А. А. Толстоногов
}

\title{
Существование оптимального управления без предположения выпуклости в эволюционной системе первого порядка
}

\begin{abstract}
Доказьвается теорема существования в задаче оптимального управления с ограничениями, описываемой линейньм эволюционньм уравнением первого порядка. Мы не делаем общепринятых предположений выпуклости, относящихся к управлению. Резултат получен путем использования свойств многозначного интеграла и условий квазивогнутости, относящихся к переменной состояния. Подробно рассмотрен пример управляемой параболической системы.

Библиографиял: 20 названий.
\end{abstract}

\section{Введение и постановка задачи}

Проблема существования оптимального управления занимает принципиальное место в теории оптимальных процессов. Как отмечает Л. Янг [1], теория необходимых условий оптимальности является наивной, если не выяснен вопрос о сушествовании решения вариационной задачи в заданном классе допустимых функций. Историю проблемы и существующие подходы к ее решению можно найти в приложении к обзору [2]. В подавляющем большинстве публикаций, посвященных существованию оптимального управления в системах, описываемых уравнениями в частных производных или эволюционными уравнениями, рассматриваются выпуклые задачи (см., например, [3]-[7] и др.). Термин "вьпуклость" подразумевает, что множество допустимых управлений является выпуклым и минимизируемый функционал является выпуклой по управлению функцией. При изучении подобных задач, как правило, используется метод Лебега-Тонелли, который состоит в доказательстве полунепрерывности снизу минимизируемого функционала на компактном множестве допустимых кривых. В конечном итоге доказательство существования решения задачи сводится к применению классической теоремы Вейерштрасса в соответствующих функциональных пространствах.

К невыпуклым относятся задачи, в которых множество допустимых управлений не является выпуклым, а минимизируемый функционал не является выпуклой по управлению функцией. Невыпуклые задачи оптимального управления, описываемые уравнениями в частных производных, изучались в работах [8]-[12]. Поскольку не существует достаточно общих схем для изучения таких задач, то в каждой из указанных выше работ рассматриваются конкретные управляемые системы с конкретным видом минимизируемого функционала и с конкретньми ограничениями.

Работа выполнена при частичной поддержке Российского фонда фундаментальных исследований (грант № 99-01-00216).

$$
\text { (C) А. А. Толстоногов } 2001
$$


Следует отметить, что подавляющее большинство результатов по невыпуклым задачам было получено путем использования различных версий теоремы Ляпунова о выпуклости и замкнутости множества значений неатомической меры в конечномерном пространстве или ее следствия о выпуклости и замкнутости многозначного интеграла (см., например, [3]).

В настоящей работе рассматривается следующая задача.

Пусть $T=[0,1], \mathbb{R}=(-\infty, \infty), \overline{\mathbb{R}}=[-\infty, \infty], \Omega \subset \mathbb{R}^{N}$ - ограниченная область в пространстве $\mathbb{R}^{N}, H=L^{2}(\Omega)$ и $V$ - сепарабельное рефлексивное банахово пространство, которое плотно, непрерьвно и компактно вложено в $H$. Отождествляя $H$ с его топологически сопряженным, мы имеем $V \hookrightarrow H \hookrightarrow V^{*}$, где $V^{*}-$ пространство, топологически сопряженное к $V$. При этом все вложения являются непрерывными, плотными и компактными. Такая тройка пространств обычно называется әволючионной или тройкой Гельфанда.

Пусть $\mathscr{L}\left(V, V^{*}\right)$ - пространство непрерьвных линейных операторов из $V$ в $V^{*}$. Как обычно, пространство $\mathscr{H}=L^{2}\left(T, L^{2}(\Omega)\right)$ мы отождествляем с пространством $L^{2}(T \times \Omega)$, используя для элемента $u \in \mathscr{H}$ представление $u(t)(z)=u(t, z), t \in T$, $z \in \Omega$. Всюду в дальнейшем сокрашение п.в. означает почти всюду.

Рассмотрим управляемую систему

$$
\begin{array}{ll}
\dot{x}(t)+A(t) x(t)=u(t) & \text { п.в., } \\
x(0)=x_{0} \in H, & u \in \mathscr{H},
\end{array}
$$

с ограничениями на управление

$$
u(t)(z)=u(t, z) \in U(t, z) \text { п.в. }
$$

где $A(t) \in \mathscr{L}\left(V, V^{*}\right)$, а $U: T \times \Omega \rightarrow \mathbb{R}$ является многозначным отображением с замкнутыми, не обязательно выпуклыми значениями.

Мы изучаем задачу $(P)$ минимизации функционала

$$
J(x, u)=G(x)+F(u)
$$

на решениях управляемой системы $(0.1),(0.2)$ при ограничениях

$$
\begin{aligned}
\Phi_{i}(x) \leqslant b_{i}, & i=1, \ldots, k, \\
Q_{j}(u) \leqslant d_{j}, & j=1, \ldots, l,
\end{aligned}
$$

где

$$
F(u)=\int_{T} \int_{\Omega} f(t, z, u(t, z)) d z d t .
$$

Здесь $G: C(T, H) \rightarrow \overline{\mathbb{R}}, \Phi_{i}: C(T, H) \rightarrow \overline{\mathbb{R}}, Q_{j}: \mathscr{H} \rightarrow \overline{\mathbb{R}}$ являются квазивогнутыми функциями, а функция $f: T \times \Omega \times \mathbb{R} \rightarrow \overline{\mathbb{R}}$ является невыпуклым нормальным интегрантом (см. [13; гл. $8, \S 1$, определение 1.1$])$.

При определенных предположениях о полунепрерывности снизу соответствующих функций и условиях их роста мы доказываем, что задача $(P)$ имеет решение.

Работа состоит из введения и пяти параграфов.

Во введении дается постановка задачи. 
В $\S 1$ вводятся основные обозначения, предположения и формулируется основной результат.

В $\S 2$ доказьвается ряд вспомогательных результатов, которые используются при доказательстве основной теоремы.

В $\S 3$ рассматривается овыпукленная, так называемая релаксационная, задача и доказывается существование решения этой задачи.

Четвертый параграф̆ посвящен доказательству основного результата.

В $\S 5$ рассматривается пример управляемой параболической системы.

\section{$\S 1$. Основной результат}

Пусть $\left(V, H, V^{*}\right)$ - эволюционная тройка пространств с нормами $\|\cdot\|,|\cdot|,\|\cdot\|_{*} \cdot$ Под $\left\langle x, x^{\prime}\right\rangle, x \in V, x^{\prime} \in V^{*}$, мы понимаем каноническую билинейную форму, устанавливающую двойственность между $V$ и $V^{*}$, a $(\cdot, \cdot)$ означает скалярное произведение в $H$. Мы считаем, что форма $\langle\cdot, \cdot\rangle$ и скалярное произведение $(\cdot, \cdot)$ согласованы, т.е. сужение $\langle\cdot, \cdot\rangle$ на $V \times H$ совпадает с $(\cdot, \cdot)$.

Введем следующие пространства: $\mathscr{V}=L^{2}(T, V), \mathscr{H}=L^{2}(T, H), \mathscr{V}^{*}=$ $L^{2}\left(T, V^{*}\right)$ и $W=\left\{\nu \in \mathscr{V} ; \dot{\nu} \in \mathscr{V}^{*}\right\}$, где производная $\dot{\nu}$ понимается в смысле векторных распределений.

Пространство $W$ с нормой $\|\nu\|=\left(\|\nu\|_{\mathscr{V}}^{2}+\|\dot{\nu}\|_{\mathscr{V} *}^{2}\right)^{1 / 2}$ является сепарабельньм рефлексивным банаховьм пространством и $W \hookrightarrow \mathscr{V} \hookrightarrow \mathscr{H} \hookrightarrow \mathscr{V}^{*}$. Известно, что любой элемент $\nu \in W$ после изменения на множестве меры нуль можно отождествить с элементом пространства $C(T, H)$. При этом вложение $W \hookrightarrow C(T, H)$ является непрерьвньм, а вложение $W \hookrightarrow \mathscr{H}$ является компактным [14].

Пусть $Z$ - отделимое локально выпуклое пространство (о.л.в.п.). Функция $g: Z \rightarrow \overline{\mathbb{R}}$ называется квазивогнутой, если для любых $z_{1}, z_{2} \in Z, 0 \leqslant \lambda \leqslant 1$ имеет место неравенство

$$
g\left(\lambda z_{1}+(1-\lambda) z_{2}\right) \geqslant \min \left(g\left(z_{1}\right), g\left(z_{2}\right)\right) .
$$

Многозначное отображение $U: T \times \Omega \rightarrow \mathbb{R}$ называется измеримылм, если для любого замкнутого множества $C \subset \mathbb{R}$ множество $\{(t, z) \in T \times \Omega ; U(t, z) \cap C \neq \varnothing\}$ измеримо.

Сделаем следующие предположения.

ГиПотеЗЫ $\mathrm{H}(\mathrm{A}) . A(t) \in \mathscr{L}\left(V, V^{*}\right)$ является оператором таким, что:

(1) отображение $t \rightarrow A(t) x$ измеримо для любого $x \in V$;

(2) $\langle x, A(t) x\rangle \geqslant c_{1}\|x\|^{2}, c_{1}>0$, для всех $t \in T$;

(3) $\|A(t) x\|_{*} \leqslant c_{2}\|x\|, c_{2}>0$, для всех $t \in T$.

ГипотезА $\mathrm{H}(\mathrm{U}) . U: T \times \Omega \rightarrow \mathbb{R}$ является измеримым многозначньп отображением с замкнутыми значениями.

ГипотЕЗА $\mathrm{H}(\mathrm{G}) . G: C(T, H) \rightarrow \overline{\mathbb{R}}$ является полунепрерывной снизу квазивогнутой функцией, удовлетворяющей неравенству

$$
G(x) \geqslant-\beta_{1}\|x\|_{C(T, H)}^{2}-\beta_{2}, \quad \beta_{1}, \beta_{2}>0 .
$$


ГипотезА $\mathrm{H}(\mathrm{f}) . f: T \times \Omega \times \mathbb{R} \rightarrow \overline{\mathbb{R}}$ является невыпукльм нормальным интегрантом, удовлетворяюшим неравенству

$$
f(t, z, u) \geqslant \alpha_{1}\|u\|_{\mathbb{R}}^{2}-\alpha_{2}(t, z) \text { п.в. на } T \times \Omega
$$

для любого $u \in \mathbb{R}, \alpha_{1}>0, \alpha_{2}(\cdot) \in L^{1}\left(T, \mathbb{R}^{+}\right)$.

ГипотеЗА $\mathrm{H}(\Phi) . \Phi_{i}: C(T, H) \rightarrow \overline{\mathbb{R}}, i=1, \ldots, k$, является полунепрерывной снизу квазивогнутой функцией.

ГипотезА $\mathrm{H}(\mathrm{Q}) . Q_{j}: \mathscr{H} \rightarrow \overline{\mathbb{R}}, j=1, \ldots, l$, является квазивогнутой функцией, полунепрерывной снизу в слабой топологии пространства $\mathscr{H}$.

Хорошо известно [14], что в рамках гипотез $\mathrm{H}(\mathrm{A})$ для любого $u \in \mathscr{H}$ уравнение (0.1) имеет единственное решение $x \in W \hookrightarrow C(T, H)$.

Под решением управляемой системь $(0.1),(0.2)$ мы понимаем пару $(x, u) \in$ $W \times \mathscr{H} \hookrightarrow C(T, H) \times \mathscr{H}$, удовлетворяюшую соотношениям $(0.1),(0.2)$.

Пару $(x, u) \in W \times \mathscr{H} \hookrightarrow C(T, H) \times \mathscr{H}$ назовем допустимой для задачи $(P)$, если она является решением управляемой системы $(0.1),(0.2)$ и удовлетворяет ограничениям $(0.4),(0.5)$.

Сделаем еше одно предположение.

ГипотезА Н $(\mathrm{J})$. Сушествует допустимая пара $\left(x^{*}, u^{*}\right)$ задачи $(P)$ такая, что

$$
J\left(x^{*}, u^{*}\right)<\infty
$$

Всюду в дальнейшем мы считаем, что гипотезы $\mathrm{H}(\mathrm{A}), \mathrm{H}(\mathrm{U}), \mathrm{H}(\mathrm{G}), \mathrm{H}(\mathrm{f}), \mathrm{H}(\Phi)$, $\mathrm{H}(\mathrm{Q}), \mathrm{H}(\mathrm{J})$ имеют место.

Основная теорема. Если $\alpha_{1}>2 \beta_{1}$, то задача $(P)$ имеет решение.

ЗАмечАниЕ. Примером функции $Q_{j}$ может служить функция

$$
Q_{j}(u)=\min _{1 \leqslant i \leqslant m_{j}}\left\{q_{j i}(u)+r_{j i}\right\}, \quad r_{j i} \in \mathbb{R},
$$

где $q_{j i}(\cdot)$ - непрерывный линейный функционал на пространстве $\mathscr{H}$.

\section{§2. Вспомогательные результаты}

Пусть $Z$ - отделимое локально выпуклое пространство, $Z^{*}$ - его топологическое и сопряженное и $\left\langle z, z^{\prime}\right\rangle_{Z}-$ каноническая билинейная форма, устанавливающая двойственность между $Z$ и $Z^{*}$.

Лемма 2.1. Пусть $g: Z \rightarrow \overline{\mathbb{R}}$ - полунепрерывная снизу квазивогнутая функция. Тогда для любого әлемента $z_{*} \in Z$ существует әлемент $z_{*}^{\prime} \in Z^{*}$ maкой, что

$$
g\left(z_{*}\right)=\sup \left\{g(z) ;\left\langle z, z_{*}^{\prime}\right\rangle_{Z}=\left\langle z_{*}, z_{*}^{\prime}\right\rangle_{Z}\right\} .
$$


ДокАЗАТЕЛЬство. Возьмем произвольньй фиксированный элемент $z_{*} \in Z$. Тогда очевидно, что для любого элемента $z^{\prime} \in Z^{*}$ справедливо неравенство

$$
g\left(z_{*}\right) \leqslant \sup \left\{g(z) ;\left\langle z, z^{\prime}\right\rangle_{Z}=\left\langle z_{*}, z^{\prime}\right\rangle_{Z}\right\}
$$

Если

$$
g\left(z_{*}\right)=\sup \{g(z) ; z \in Z\},
$$

то равенство (2.1) имеет место при любом $z_{*}^{\prime} \in Z^{*}$.

Предположим, что

$$
g\left(z_{*}\right)<\sup \{g(z) ; z \in Z\} .
$$

Из неравенства (1.1) вытекает, что для любого $m \leqslant \sup \{g(z) ; z \in Z\}$ множество $\{z \in Z ; g(z)>m\}$ является непустьм и выпуклым. Тогда, воспользовавшись полунепрерывностью снизу функции $g(z)$, получаем, что множество

$$
L=\left\{z \in Z ; g(z)>g\left(z_{*}\right)\right\}
$$

является непустым, выпуклым, открытым и $z_{*} \notin L$. Следовательно, согласно следствию 1.1 из [13; гл. $1, \S 1]$ существует элемент $z_{*}^{\prime} \in Z^{*}$ такой, что

$$
\left\langle z, z_{*}^{\prime}\right\rangle_{Z}>\left\langle z_{*}, z_{*}^{\prime}\right\rangle_{Z} \quad \forall z \in L
$$

Поэтому

$$
g(z) \leqslant g\left(z_{*}\right)
$$

для любого $z \in Z$, удовлетворяющего неравенству

$$
\left\langle z, z_{*}^{\prime}\right\rangle_{Z} \leqslant\left\langle z_{*}, z_{*}^{\prime}\right\rangle_{Z}
$$

Теперь справедливость равенства (2.1) вытекает из неравенств (2.2), (2.3). Лемма доказана.

Пусть $X$ - о.л.в.п. Оператор $P: X \rightarrow Z$ называется аффинным, если

$$
P x=z_{0}+P_{1} x
$$

где $z_{0} \in Z$ - некоторый фиксированньй элемент, а $P: X \rightarrow Z$ - линейньй оператор.

Очевидно, что если $P: X \rightarrow Z$ - аффинный оператор, а $g: Z \rightarrow \overline{\mathbb{R}}$ - квазивогнутая функция, то функция $\widetilde{g}: X \rightarrow \overline{\mathbb{R}}, \widetilde{g}(x)=g(P x), \quad x \in X$, является квазивогнутой.

Пусть $B_{\mathscr{H}}(r)=\left\{u \in \mathscr{H} ;\|u\|_{\mathscr{H}} \leqslant r\right\}, r>0$, и $\mathscr{R}_{r}\left(x_{0}\right)-$ совокупность всех решений уравнения $(0.1)$ с $u \in B_{\mathscr{H}}(r)$.

Лемма 2.2. Существуют $M_{i}>0, i=0, \ldots, 4$, такие, что для любого $x \in \mathscr{R}_{r}\left(x_{0}\right)$ справедливь оценки

$$
\begin{aligned}
|x(t)| & \leqslant M_{1}, \quad t \in T, \\
\|x\|_{\mathscr{V}} & \leqslant M_{2}, \\
\|\dot{x}\|_{\mathscr{V}^{*}} & \leqslant M_{3}, \\
\|x\|_{W} & \leqslant M_{4} .
\end{aligned}
$$


ДокАЗАТЕЛЬСтво. Пусть $x \in \mathscr{R}_{r}\left(x_{0}\right)$ - решение уравнения $(0.1)$, соответствующее $u \in B \mathscr{H}(r)$. Тогда

$$
\langle x(t), \dot{x}(t)\rangle+\langle x(t), A(t) x(t)\rangle=(x(t), u(t)) \text { п.в. }
$$

Из этого равенства и гипотезы Н(А) (2) следует

$$
\frac{1}{2} \frac{d}{d t}|x(t)|^{2}+c_{1}\|x(t)\|^{2} \leqslant|u(t)| \cdot|x(t)| \text { п.в. }
$$

Так как $V$ вложено в $H$ непрерывно, то существует $\gamma>0$ такое, что $|\cdot| \leqslant \gamma\|\cdot\|$. Воспользовавшись неравенством Коши для $\varepsilon>0$, мы получим

$$
|f(t)| \cdot|x(t)| \leqslant \gamma|u(t)| \cdot\|x(t)\| \leqslant \frac{\varepsilon^{2} \gamma}{2}|u(t)|^{2}+\frac{\gamma}{2 \varepsilon^{2}}\|x(t)\|^{2} \text { п.в. }
$$

Выберем $\varepsilon>0$ так, чтобы $2 \varepsilon^{2}=\gamma / c_{1}$. Тогда из $(2.8),(2.9)$ вытекает

$$
\frac{1}{2} \frac{d}{d t}|x(t)|^{2} \leqslant \frac{\gamma^{2}}{4 c_{1}}|u(t)|^{2} \text { п.в. }
$$

Интегрируя (2.10), мы получаем

$$
|x(t)|^{2} \leqslant \frac{\gamma^{2}}{2 c_{1}}\|u\|_{\mathscr{H}}^{2}+2|x(0)| .
$$

Следовательно, существует $M_{1}>0$ такое, что справедливо неравенство (2.4).

Используя это неравенство и (2.8), мы приходим к неравенству

$$
2 c_{1} \int_{0}^{t}\|x(s)\|^{2} d s \leqslant 2 M_{1} \int_{0}^{t}|u(s)| d s+2|x(0)|^{2} .
$$

Отсюда вытекает, что существует $M_{2}>0$ такое, что справедливо неравенство $(2.5)$.

Пусть $h \in \mathscr{V}$. Тогда

$$
\begin{aligned}
\int_{0}^{t}\langle h(s), \dot{x}(s)\rangle d s & =\int_{0}^{t}\langle-h(s), A(s) x(s)\rangle d s+\int_{0}^{t}(h(s), u(s)) d s \\
& \leqslant \int_{0}^{t}\|A(s) x(s)\|_{*} \cdot\|h(s)\| d s+\gamma \int_{0}^{t}|u(s)| \cdot\|h(s)\| d s \\
& \leqslant \int_{0}^{t} c_{2}\|x(s)\| \cdot\|h(s)\| d s+\gamma \int_{0}^{t}|u(s)| \cdot\|h(s)\| d s .
\end{aligned}
$$

Используя это неравенство, неравенство (2.5) и неравенство Гёльдера, мы получаем, что сушествует $M_{3}>0$ такое, что

$$
\int_{0}^{t}\langle h(s), \dot{x}(s)\rangle d s \leqslant M_{3}\|h\|_{\mathscr{V}}
$$

Отсюда вытекает неравенство (2.6).

Неравенство (2.7) является следствием неравенств (2.6), (2.7). Лемма доказана.

В дальнейшем для любого банахова пространства $X$ символ $w$ - $X$ означает, что пространство $X$ наделено слабой $\sigma\left(X, X^{*}\right)$ топологией [15]. Во всех остальных случаях мы считаем, что пространство $X$ наделено сильной (нормированной) топологией. 
Лемма 2.3. Пусть $P: \mathscr{H} \rightarrow C(T, H)$ - оператор, который кажсдому әлементу $и \in \mathscr{H}$ ставит в соответствие единственное решение $x=P$ уравнения (0.1). Тогда $P$ является аффинным, т.е. $P u=y_{0}+P_{1} u$, дде $y_{0} \in C(T, H)$ - фиксированный элемент, а $P_{1}: \mathscr{H} \rightarrow C(T, H)$ является линейным компактнымм нерастягивающим оператором.

ДоказАтельство. Пусть $y_{0} \in W \hookrightarrow C(T, H)$ - единственное решение уравнения $(0.1)$, соответствуюшее $u=\Theta_{\mathscr{H}}$, где $\Theta \mathscr{H}-$ нулевой элемент пространства $\mathscr{H}$, и $y=P_{1} u$ - единственное решение $y \in W \hookrightarrow C(T, H)$ уравнения $(0.1)$ с $x(0)=\Theta_{H}$, где $\Theta_{H}$ - нулевой элемент пространства $H$. Тогда, очевидно, $P_{1}: \mathscr{H} \rightarrow C(T, H)$ является линейньм оператором и любое решение $x$ уравнения $(0.1)$ представимо в виде $x=P u=y_{0}+P_{1} u$.

Докажем компактность оператора $P_{1}$. Пусть $B_{\mathscr{H}}(1)$ - замкнутый единичный шар пространства $\mathscr{H}$. Так как $B_{\mathscr{H}}(1)$ является метризуемьм компактнымподмножеством пространства $w$ - $\mathscr{H}$, то нам достаточно показать, что если $u_{n} \in B_{\mathscr{H}}(1)$ сходится к $u$ в пространстве $w$ - $\mathscr{H}$, то последовательность $x_{n}=P_{1} u_{n}$ сходится к $x=P_{1} u$ в пространстве $C(T, H)$.

Пусть $u_{n}, u \in B_{\mathscr{H}}(1), n \geqslant 1$, таковы, что

$$
u_{n} \rightarrow u \quad \text { в } w-\mathscr{H}
$$

и $x_{n}=P_{1} u_{n}, x=P_{1} u$. Из леммы 2.2 следует, что последовательность $x_{n}, n \geqslant 1$, ограничена в пространстве $W$ и, следовательно, является относительно компактным метризуемым подмножеством пространства $w$ - $W$. Переходя к подпоследовательности, если необходимо, мы можем предположить, что

$$
x_{n} \rightarrow y \quad \text { в } \quad w-W
$$

для некоторого $y \in W$. Так как вложение $W \hookrightarrow \mathscr{H}$ компактно, то

$$
x_{n} \rightarrow y \quad \text { в } \mathscr{H}
$$

Покажем, что $x=y$. Используя монотонность оператора $A(t)$ (см. гипотезу $\mathrm{H}(\mathrm{A})(2))$ и уравнение $(0.1)$, мы получаем, что

$$
\begin{aligned}
\frac{1}{2}\left|x_{n}(t)-x(t)\right|^{2} \leqslant & \int_{0}^{t}\left(x_{n}(s)-x(s), u_{n}(s)-u(s)\right) d s \\
\leqslant & \int_{0}^{t}\left(x_{n}(s)-y(s), u_{n}(s)-u(s)\right) d s \\
& +\int_{0}^{t}\left(y(s)-x(s), u_{n}(s)-u(s)\right) d s .
\end{aligned}
$$

Из последнего неравенства в (2.14) и (2.11), (2.13) вытекает, что

$$
x_{n}(t) \rightarrow x(t) \quad \text { в } H, \quad t \in T .
$$


Воспользовавшись $(2.4),(2.13),(2.15)$, мы получим, что $x=y$. Следовательно, в соответствии с (2.13)

$$
x_{n} \rightarrow x \quad \text { в } \mathscr{H}
$$

Учитывая, что уравнение (0.1) имеет единственное решение, и имея в виду первое неравенство в (2.14), а также (2.11), (2.16) и неравенство Гёльдера, мы заключаем, что вся последовательность $x_{n}, n \geqslant 1$, сходится к $x$ в $C(T, H)$. Следовательно, оператор $P_{1}: \mathscr{H} \rightarrow C(T, H)$ компактен.

Пусть $u \in \mathscr{H}$ и $x=P_{1} u$. По аналогии с неравенством (2.14) мы получаем

$$
\frac{1}{2}|x(t)|^{2} \leqslant \int_{0}^{t}|u(s)| \cdot|x(s)| d s .
$$

Воспользовавшись леммой А.5 из [16], мы приходим к неравенству

$$
|x(t)| \leqslant \int_{0}^{t}|u(s)| d s, \quad t \in T=[0,1] .
$$

Последнее неравенство и неравенство Гёльдера нам дают, что

$$
\|x\|_{C(T, H)}=\left\|P_{1} x\right\|_{C(T, H)} \leqslant\|u\|_{\mathscr{H}}
$$

Следовательно, оператор $P_{1}: \mathscr{H} \rightarrow C(T, H)$ является нерастягивающим. Лемма доказана.

\section{§3. Релаксационная задача}

В этом параграфе мы рассмотрим вспомогательную, так называемую релаксационную, задачу.

Пусть $\widetilde{f}: T \times \Omega \times \mathbb{R} \rightarrow \overline{\mathbb{R}}-$ функция, определенная следующим образом:

$$
\tilde{f}(t, z, u)= \begin{cases}f(t, z, u), & u \in U(t, z), \\ +\infty, & u \notin U(t, z)\end{cases}
$$

и $f^{* *}(t, z, u)$ - биполяра [13; гл. $\left.1, \S 4\right]$ функции $u \rightarrow \widetilde{f}(t, z, u)$.

УТВЕРЖДЕНИЕ 3.1. Функиия $f^{* *}(t, z, u)$ является нормальным интегранmoм $u$

$$
\alpha_{1}\|u\|_{\mathbb{R}}^{2}-\alpha_{2}(t, z) \leqslant f^{* *}(t, z, u) \leqslant \widetilde{f}(t, z, u) \quad \text { n.в. }
$$

ДоказАТЕЛьство. Пусть $\mu_{0}$ - мера Лебега на $T \times \Omega$. Из [17; лемма 1.3] и $[13 ;$ гл. $8, \S 1$, теорема 1.1] следует, что для любого $\varepsilon>0$ сушествует компакт $Q_{\varepsilon} \subset T \times \Omega$ такой, что $\mu_{0}\left((T \times \Omega) \backslash Q_{\varepsilon}\right) \leqslant \varepsilon$ и сужение $f(t, z, u)$ на $Q_{\varepsilon} \times \mathbb{R}$ является полунепрерывной снизу функцией, а сужение $U(t, z)$ на $Q_{\varepsilon}$ имеет замкнутый график в $Q_{\varepsilon} \times \mathbb{R}$. Тогда согласно (3.1) сужение $\tilde{f}(t, z, u)$ на $Q_{\varepsilon} \times \mathbb{R}$ будет полунепрерывной снизу функцией. Воспользовавшись теоремой 1.1 из [13; гл. $8, \S 1]$, получим, что функция $\widetilde{f}(t, z, u)$ является нормальным интегрантом. Тогда из [13; гл. $8, \S 1$, предложение 1.3] вытекает, что функция $f^{* *}(t, z, u)$ также является нормальным интегрантом. Так как функция $u \rightarrow \alpha_{1}\|u\|_{\mathbb{R}}^{2}-\alpha_{2}(t, z)$ является выпуклой, 
то, воспользовавшись (1.3), (3.1) и предложением 4.1 из [13; гл. $1, \S 4]$, мы приходим к неравенству (3.2). Утверждение доказано.

Рассмотрим релаксационную задачу $(R P)$ минимизации функционала

$$
J^{* *}(x, u)=G(x)+F^{* *}(u)
$$

на решениях управляемой системы, описываемой уравнением (0.1) с ограничениями на управление

$$
u(t)(z)=u(t, z) \in \overline{\mathrm{co}} U(t, z) \text { п.в. }
$$

и ограничениями $(0.4),(0.5)$, где

$$
F^{* *}(u)=\int_{T} \int_{\Omega} f^{* *}(t, z, u(t, z)) d z d t,
$$

а $\overline{\mathrm{co}} U(t, z)$ означает замкнутую выпуклую оболочку множества $U(t, z)$.

Теорема 3.1. Если $\alpha_{1}>2 \beta_{1}$, то задача $(R P)$ имеет решение.

ДокАЗАТЕльство. Из утверждения 3.1 следует, что для любого $u \in \mathscr{H}$ функция $(t, z) \rightarrow f^{* *}(t, z, u(t, z))$ измерима. Поэтому в соответствии с $(1.2),(3.2)$ для любого решения $(x, u)$ управляемой системы $(0.1),(3.3)$ имеет место неравенство

$$
-\infty<J^{* *}(x, u) \leqslant+\infty
$$

Пусть $\left(x_{n}, u_{n}\right), n \geqslant 1,-$ минимизирующая последовательность задачи $(R P)$. Если $\left(x^{*}, u^{*}\right)$ - допустимая пара задачи $(P)$, то из $(3.3)$ следует, что $\left(x^{*}, u^{*}\right)$ является допустимой парой и для задачи $(R P)$. Воспользовавшись неравенствами (1.4), (3.4), мы получим, что существует $M>0$ такое, что

$$
-\infty<J^{* *}\left(x_{n}, u_{n}\right)<M, \quad n \geqslant 1 \text {. }
$$

Следовательно, в соответствии с (1.2), (3.2)

$$
-\beta_{2}-\beta_{1}\left\|x_{n}\right\|_{C(T, H)}^{2}+\alpha_{1}\left\|u_{n}\right\|_{\mathscr{H}}^{2}-\alpha_{2} \leqslant M
$$

где $\alpha_{2}=\int_{T} \int_{\Omega} \alpha_{2}(t, z) d z d t$.

Из (3.5) и леммы 2.3 вытекает

$$
\begin{aligned}
& -\beta_{2}-2 \beta_{1}\left\|y_{0}\right\|_{C(T, H)}^{2}-2 \beta_{1}\left\|u_{n}\right\|_{\mathscr{H}}^{2}+\alpha_{1}\left\|u_{n}\right\|_{\mathscr{H}}^{2}-\alpha_{2} \\
& \quad \leqslant-\beta_{2}-\beta_{1}\left\|y_{0}+P_{1} u_{n}\right\|_{C(T, H)}^{2}+\alpha_{1}\left\|u_{n}\right\|_{\mathscr{H}}^{2}-\alpha_{2} \leqslant M .
\end{aligned}
$$

Так как $\alpha_{1}-2 \beta_{1}>0$, то из неравенства (3.6) следует, что последовательность $u_{n}$, $n \geqslant 1$, ограничена в пространстве $\mathscr{H}$. Так как $\mathscr{H}$ является рефлексивным пространством, то, не нарушая общности, мы можем считать, что последовательность $u_{n}, n \geqslant 1$, сходится к некоторому элементу $u_{*}$ в пространстве $w$ - $\mathscr{H}$. Хорошо известно, что в этом случае

$$
u_{*}(t, z) \in \bigcap_{n=1}^{\infty} \overline{\mathrm{co}} \bigcup_{k \geqslant n}^{\infty} u_{k}(t, z) \in \overline{\mathrm{co}} U(t, z) \text { п.в. }
$$


Воспользовавшись леммой 2.3, мы получим, что последовательность решений $x_{n}=P u_{n}, n \geqslant 1$, уравнения (0.1) сходится в пространстве $C(T, H)$ к решению $x_{*}=P u_{*}$ уравнения (0.1). Следовательно, в соответствии с $(3.7)$ пара $\left(x_{*}, u_{*}\right)$ является решением управляемой системы $(0.1),(3.3)$.

Из гипотез $\mathrm{H}(\Phi), \mathrm{H}(\mathrm{Q})$, сходимости $x_{n}, n \geqslant 1, \mathrm{\kappa} x_{*}$ в $C(T, H)$, сходимости $u_{n}$, $n \geqslant 1, \mathrm{k} u_{*}$ в $w$ - $\mathscr{H}$ следует, что

$$
\begin{aligned}
\Phi_{i}\left(x_{*}\right) \leqslant b_{i}, & i=1, \ldots, k, \\
Q_{j}\left(u_{*}\right) \leqslant d_{j}, & j=1, \ldots, l .
\end{aligned}
$$

Воспользовавшись леммой 2.3, утверждением 3.1 и следствием 1.2 из [13; гл. $8, \S 1]$, получаем, что функция $u \rightarrow G(P u)+F^{* *}(u)$ секвенциально полунепрерьвна снизу в топологии пространства $w$ - $\mathscr{H}$. Поэтому

$$
J^{* *}\left(x_{*}, u_{*}\right) \leqslant \lim _{n \rightarrow \infty} J^{* *}\left(x_{n}, u_{n}\right) .
$$

Принимая во внимание (3.7)-(3.10), мы получаем, что пара $\left(x_{*}, u_{*}\right)$ является решением задачи $(R P)$. Теорема доказана.

\section{§4. Доказательство основной теоремы}

Рассмотрим задачу $(\widetilde{P})$ минимизации функционала

$$
\widetilde{J}(x, u)=G(x)+\widetilde{F}(u)
$$

на решениях управляемой системы $(0.1),(0.2)$ с ограничениями $(0.4),(0.5)$, где

$$
\widetilde{F}(u)=\int_{T} \int_{\Omega} \widetilde{f}(t, z, u(t, z)) d z d t .
$$

Из (3.1) следует, что любая допустимая пара $(x, u)$ задачи $(P)$ является допустимой и для задачи $(\widetilde{P})$. Тогда согласно $(3.2)$

$$
\min J^{* *}(x, u) \leqslant \inf \widetilde{J}(x, u)<\infty
$$

и, принимая во внимание (3.1), мы получаем, что задачи $(P)$ и $(\widetilde{P})$ эквивалентны.

Пусть $\left(x_{*}, u_{*}\right)$ - решение задачи $(R P)$. Согласно (4.1) теорема будет доказана, если мы покажем, что существует допустимая пара $\left(x_{0}, u_{0}\right)$ задачи $(\widetilde{P})$ такая, что

$$
J^{* *}\left(x_{*}, u_{*}\right)=\widetilde{J}\left(x_{0}, u_{0}\right) .
$$

Из (1.2), (3.2) следует, что

$$
-\infty<\int_{T} \int_{\Omega} f^{* *}\left(t, z, u_{*}(t, z)\right) d z d t<\infty
$$

Поэтому функция $f^{* *}\left(t, z, u_{*}(t, z)\right)$ интегрируема и, следовательно,

$$
\left|f^{* *}\left(t, z, u_{*}(t, z)\right)\right|<\infty \text { п.в. }
$$


Воспользовавшись предложением 3.1 из [13; гл. $9, \S 3]$, мы получаем, что существуют измеримые функции $p_{i}: T \times \Omega \rightarrow \mathbb{R}^{+}, u_{i}: T \times \Omega \rightarrow \mathbb{R}, i=1,2$, такие, что

$$
\begin{gathered}
u_{*}(t, z)=\sum_{i=1}^{2} p_{i}(t, z) u_{i}(t, z) \text { п.в., } \\
f^{* *}\left(t, z, u_{*}(t, z)\right)=\sum_{i=1}^{2} p_{i}(t, z) \widetilde{f}\left(t, z, u_{i}(t, z)\right) \text { п.в. } \\
\sum_{i=1}^{2} p_{i}(t, z)=1 \text { п.в. }
\end{gathered}
$$

Положим

$$
E_{i}=\left\{(t, z) \in T \times \Omega ; u_{i}(t, z) \notin U(t, z)\right\}, \quad i=1,2 .
$$

Воспользовавшись утверждением е) теоремы 3.5 из [18], мы получим, что множества $E_{i}, i=1,2$, измеримы. Если $(t, z) \in E_{i}$ и $\mu_{0}\left(E_{i}\right)=0$, то $u_{i}(t, z) \in U(t, z)$ п.в. на $T \times \Omega$. Если же $\mu_{0}\left(E_{i}\right)>0$, то в соответствии с $(3.1) \tilde{f}\left(t, z, u_{i}(t, z)\right)=\infty$ п.в. на $E_{i}$. Тогда из $(4.2),(4.4),(4.5)$ вытекает, что $p_{i}(t, z)=0$ п.в. на $E_{i}$. Следовательно, не нарушая равенств $(4.3),(4.4)$, мы можем изменить $u_{i}(t, z)$ на множестве $E_{i}$ так, что $u_{i}(t, z)$ останутся измеримьми функциями и

$$
u_{i}(t, z) \in U(t, z), \quad i=1,2, \quad \text { п.в. на } T \times \Omega \text {. }
$$

Тогда в соответствии с (3.1), (4.4), (4.6)

$$
f^{* *}\left(t, z, u_{*}(t, z)\right)=\sum_{i=1}^{2} p_{i}(t, z) f\left(t, z, u_{i}(t, z)\right) \text { п.в. }
$$

Воспользовавшись (4.3), (4.5), (4.6), мы получим

$$
u_{*}(t, z) \in \operatorname{co} U(t, z) \text { п.в. }
$$

где со $U(t, z)$ означает вьпуклую оболочку множества $U(t, z)$.

Из теоремы Лузина $[13 ;$ гл. $8, \S 1]$ следует, что существует последовательность $Q_{l}, l=1,2, \ldots, Q_{l} \subset T \times \Omega$, попарно непересекающихся компактных множеств таких, что $\mu_{0}\left(\bigcup_{l=1}^{\infty} Q_{l}\right)=\mu_{0}(T \times \Omega)$ и сужение каждой из функций $u_{i}(t, z)$, $f\left(t, z, u_{i}(t, z)\right), i=1,2$, на любое из множеств $Q_{l}$ непрерьвно.

Рассмотрим измеримое многозначное отображение $\Gamma: T \times \Omega \rightarrow \mathbb{R}$ с компактными значениями, определенное следующим образом

$$
\Gamma(t, z)=\bigcup_{i=1}^{2} f\left(t, z, u_{i}(t, z)\right)
$$

Тогда из (4.7) вытекает, что

$$
f^{* *}\left(t, z, u_{*}(t, z)\right) \in \operatorname{co} \Gamma(t, z) \text { п.в. }
$$


Так как функции $f\left(t, z, u_{i}(t, z)\right), i=1,2$, непрерывны на каждом из множеств $Q_{l}$, $i=1,2, \ldots$, то многозначное отображение $\Gamma(t, z)$ интегрируемо на каждом из множеств $Q_{l}$. Воспользовавшись свойствами многозначного интеграла в конечномерном пространстве [19], мы получим

$$
\iint_{Q_{l}} \Gamma(t, z) d z d t=\iint_{Q_{l}} \operatorname{co} \Gamma(t, z) d z d t
$$

Из этого равенства и (4.9) следует, что для каждого $l=1,2, \ldots$ сушествуют попарно непересекаюшиеся множества $Q_{l}^{i}, i=1,2$, такие, что $\mu_{0}\left(\bigcup_{l=1}^{2} Q_{l}^{i}\right)=$ $\mu_{0}\left(Q_{l}\right)$ и

$$
\begin{aligned}
\iint_{Q_{l}} f^{* *}\left(t, z, u_{*}(t, z)\right) d z d t & =\iint_{Q_{l}} \sum_{i=1}^{2} p_{i}(t, z) f\left(t, z, u_{i}(t, z)\right) d z d t \\
& =\iint_{Q_{l}} \sum_{i=1}^{2} \chi\left(Q_{l}^{i}\right)(t, z) f\left(t, z, u_{i}(t, z)\right) d z d t
\end{aligned}
$$

где $\chi\left(Q_{l}^{i}\right)(t, z)$ означает характеристическую функцию множества $Q_{l}$.

Покажем, что для любого измеримого разбиения $Q_{l}^{i}, i=1,2$, множества $Q_{l}$, для которого имеет место равенство (4.10), функция

$$
g(t, z)=\sum_{l=1}^{\infty} \sum_{i=1}^{2} \chi\left(Q_{l}^{i}\right)(t, z) f\left(t, z, u_{i}(t, z)\right)
$$

интегрируема на $T \times \Omega$.

Рассмотрим последовательность

$$
g_{n}(t, z)=\sum_{l \leqslant n} \sum_{i=1}^{2} \chi\left(Q_{l}^{i}\right)(t, z)\left(f\left(t, z, u_{i}(t, z)\right)+\alpha_{2}(t, z)\right)
$$

Из (1.3) следует, что функции $g_{n}(t, z), n \geqslant 1$, неотрицательны, интегрируемы на $T \times \Omega$, последовательность $g_{n}(t, z), n \geqslant 1$, монотонно не убывает и почти всюду на $T \times \Omega$ сходится к функции $g(t, z)+\alpha_{2}(t, z)$. Воспользовавшись $(4.10),(4.12)$, получим

$$
\begin{aligned}
\int_{T} \int_{\Omega} g_{n}(t, z) d z d t & =\sum_{l \leqslant n} \iint_{Q_{l}}\left(\sum_{i=1}^{2} \chi\left(Q_{l}^{i}\right)(t, z) f\left(t, z, u_{i}(t, z)\right)+\alpha_{2}(t, z)\right) d z d t \\
& =\sum_{l \leqslant n} \iint_{Q_{l}}\left(\sum_{i=1}^{2} p_{i}(t, z) f(t, z, u(t, z))+\alpha(t, z)\right) d z d t \\
& =\sum_{l \leqslant n} \iint_{Q_{l}}\left(f^{* *}\left(t, z, u_{*}(t, z)\right)+\alpha_{2}(t, z)\right) d z d t
\end{aligned}
$$


Из (3.2) следует, что функция $f^{* *}\left(t, z, u_{*}(t, z)\right)+\alpha_{2}(t, z)$ неотрицательна. Так как функция $f^{* *}\left(t, z, u_{*}(t, z)\right)$ интегрируема на $T \times \Omega$, то из (4.13) и леммы Фату следует, что

$$
\begin{aligned}
\lim _{n \rightarrow \infty} \int_{T} \int_{\Omega} g_{n}(t, z) d z d t & =\int_{T} \int_{\Omega} \lim _{n \rightarrow \infty} g_{n}(t, z) d z d t \\
& =\int_{T} \int_{\Omega}\left(g(t, z)+\alpha_{2}(t, z)\right) d z d t<\infty
\end{aligned}
$$

Следовательно, функция $g(t, z)$ интегрируема на $T \times \Omega$.

Рассмотрим функцию

$$
u_{0}(t, z)=\sum_{l=1}^{\infty} \sum_{i=1}^{2} \chi\left(Q_{l}^{i}\right)(t, z) u_{i}(t, z)
$$

Воспользовавшись (1.3), (4.11), получим $-\alpha_{2}(t, z)-\alpha_{1}\left\|u_{0}(t, z)\right\|_{\mathbb{R}}^{2} \leqslant g(t, z)$ п.в. Отсюда следует, что $u_{0} \in \mathscr{H}, u_{0}(t, z)=u_{0}(t)(z)$. Согласно (4.6)

$$
u_{0}(t, z) \in U(t, z) \text { п.в. }
$$

Для простоты изложения и краткости обозначений будем считать, что ограничения $(0.4),(0.5)$ имеют вид

$$
\Phi(x) \leqslant b, \quad Q(u) \leqslant d,
$$

где $\Phi: C(T, H) \rightarrow \overline{\mathbb{R}}, Q: \mathscr{H} \rightarrow \overline{\mathbb{R}}$. Воспользовавшись гипотезами $\mathrm{H}(\mathrm{G}), \mathrm{H}(\Phi)$ и леммой 2.3 , получим, что функции $u \rightarrow G(P u), u \rightarrow \Phi(P u)$ являются квазивогнутыми и полунепрерывными снизу из $\mathscr{H}$ в $\overline{\mathbb{R}}$, а функция $u \rightarrow Q(u)$ является квазивогнутой и полунепрерывной снизу из $w$ - $\mathscr{H}$ в $\overline{\mathbb{R}}$. Тогда согласно лемме 2.1 существуют элементы $h_{j} \in \mathscr{H}=\mathscr{H}^{*}, j=1,2,3$, такие, что

$$
\begin{aligned}
G\left(P u_{*}\right) & =\sup \left\{G(P u) ;\left\langle u, h_{1}\right\rangle_{\mathscr{H}}=\left\langle u_{*}, h_{1}\right\rangle_{\mathscr{H}}\right\}, \\
\Phi\left(P u_{*}\right) & =\sup \left\{\Phi(P u) ;\left\langle u, h_{2}\right\rangle_{\mathscr{H}}=\left\langle u_{*}, h_{2}\right\rangle_{\mathscr{H}}\right\}, \\
Q\left(u_{*}\right) & =\sup \left\{Q(u) ;\left\langle u, h_{3}\right\rangle_{\mathscr{H}}=\left\langle u_{*}, h_{3}\right\rangle_{\mathscr{H}}\right\},
\end{aligned}
$$

где

$$
\left\langle u, h_{j}\right\rangle_{\mathscr{H}}=\int_{T} \int_{\Omega}\left\langle u(t, z), h_{j}(t, z)\right\rangle_{\mathbb{R}} d z d t, \quad j=1,2,3 .
$$

Рассмотрим измеримые функции $\gamma_{i}: T \times \Omega \rightarrow \mathbb{R}^{4}, i=1,2$,

$$
\begin{array}{r}
\gamma_{i}(t, z)=\left\{\left\langle u_{i}(t, z), h_{1}(t, z)\right\rangle_{\mathbb{R}},\left\langle u_{i}(t, z), h_{2}(t, z)\right\rangle_{\mathbb{R}}\right. \\
\left.\left\langle u_{i}(t, z), h_{3}(t, z)\right\rangle_{\mathbb{R}}, f\left(t, z, u_{i}(t, z)\right)\right\}
\end{array}
$$

где $u_{i}(t, z)$ удовлетворяют равенству (4.3).

Пусть $\Gamma^{*}: T \times \Omega \rightarrow \mathbb{R}^{4}$ - измеримое многозначное отображение с компактньми значениями, определенное следующим образом

$$
\Gamma^{*}(t, z)=\bigcup_{i=1}^{2} \gamma_{i}(t, z)
$$


Так как на каждом из множеств $Q_{l}, l=1,2, \ldots$, функции $u_{i}(t, z), f\left(t, z, u_{i}(t, z)\right)$, $i=1,2$, непрерьвны, то на каждом из множеств $Q_{l}, l=1,2, \ldots$, многозначное отображение $\Gamma^{*}(t, z)$ интегрируемо.

Рассмотрим функцию $\gamma^{*}: T \times \Omega \rightarrow \mathbb{R}^{4}$

$$
\begin{array}{r}
\gamma^{*}(t, z)=\left\{\left\langle u_{*}(t, z), h_{1}(t, z)\right\rangle_{\mathbb{R}},\left\langle u_{*}(t, z), h_{2}(t, z)\right\rangle_{\mathbb{R}},\right. \\
\left.\left\langle u_{*}(t, z), h_{3}(t, z)\right\rangle_{\mathbb{R}}, f^{* *}\left(t, z, u_{*}(t, z)\right)\right\} .
\end{array}
$$

Тогда из (4.3), (4.7), (4.21) следует, что

$$
\gamma^{*}(t, z) \in \operatorname{co} \Gamma^{*}(t, z) \text { п.в. }
$$

Так как множество $\Gamma^{*}(t, z)$ состоит из двух точек в пространстве $\mathbb{R}^{4}$, то из (4.23) и свойств многозначного интеграла следует, что для каждого $l=1,2, \ldots$ существует измеримое разбиение $Q_{l}^{i}, i=1,2$, множества $Q_{l}$ такое, что

$$
\iint_{Q_{l}} \gamma^{*}(t, z) d z d t=\iint_{Q_{l}} \sum_{i=1}^{2} \chi\left(Q_{l}^{i}\right)(t, z) \gamma_{i}(t, z) d z d t .
$$

Воспользовавшись (4.21), (4.22), (4.24), получим

$$
\begin{aligned}
& \iint_{Q_{l}}\left\langle u_{*}(t, z), h_{j}(t, z)\right\rangle_{\mathbb{R}} d z d t \\
&=\iint_{Q_{l}}\left\langle\sum_{i=1}^{2} \chi\left(Q_{l}^{i}\right)(t, z) u_{i}(t, z), h_{j}(t, z)\right\rangle_{\mathbb{R}} d z d t, \quad j=1,2,3 \\
& \iint_{Q_{l}} f^{* *}\left(t, z, u_{*}(t, z)\right) d z d t=\iint_{Q_{l}} \sum_{i=1}^{2} \chi\left(Q_{l}^{i}\right)(t, z) f\left(t, z, u_{i}(t, z)\right) d z d t \\
&=\iint_{Q_{l}} f\left(t, z, \sum_{i=1}^{2} \chi\left(Q_{l}^{i}\right)(t, z) u_{i}(t, z)\right) d z d t .
\end{aligned}
$$

Определим измеримую функцию

$$
u_{0}(t, z)=\sum_{l}^{\infty} \sum_{i=1}^{2} \chi\left(Q_{l}^{i}\right)(t, z) u_{i}(t, z) .
$$

Тогда из (4.6), (4.27) следует, что

$$
u_{0}(t, z) \in U(t, z) \text { п.в. на } T \times \Omega .
$$

Вьше было показано, что для любого измеримого разбиения $Q_{l}^{i}, i=1,2$, множества $Q_{l}$, для которого имеет место равенство (4.10), функция (4.14) является элементом пространства $\mathscr{H}$. Воспользовавшись (4.26), (4.27), получаем, что $u_{0} \in \mathscr{H}$, где $u_{0}(t)(z)=u_{0}(t, z)$. Из $(4.25),(4.26)$ вытекает

$$
\begin{aligned}
\int_{T} \int_{\Omega}\left\langle u_{0}(t, z), h_{j}(t, z)\right\rangle_{\mathbb{R}} d z d t & =\int_{T} \int_{\Omega}\left\langle u_{*}(t, z), h_{j}(t, z)\right\rangle_{\mathbb{R}} d z d t, \quad j=1,2,3, \\
\int_{T} \int_{\Omega} f^{* *}\left(t, z, u_{*}(t, z)\right) d z d t & =\int_{T} \int_{\Omega} f\left(t, z, u_{0}(t, z)\right) d z d t .
\end{aligned}
$$


Пусть $x_{0}=P u_{0}$ - решение уравнения (0.1). Тогда из (4.16)-(4.19), (4.29), (4.30) получаем

$$
\begin{aligned}
G\left(P u_{0}\right) & \leqslant G\left(P u_{*}\right), \\
\Phi\left(P u_{0}\right) & \leqslant \Phi\left(P u_{*}\right) \leqslant b, \\
Q\left(u_{0}\right) & \leqslant Q\left(u_{*}\right) \leqslant d .
\end{aligned}
$$

Воспользовавшшись (3.1), (4.1), (4.30), (4.31), мы приходим к равенству

$$
\widetilde{I}\left(x_{0}, u_{0}\right)=I\left(x_{0}, u_{0}\right)=I^{* *}\left(x_{*}, u_{*}\right) .
$$

Теперь из (4.28), (4.32)-(4.34) следует, что пара $x_{0}, u_{0}$ является решением задачи $(P)$. Основная теорема доказана.

\section{§5. Пример}

В этом параграфе мы рассмотрим задачу оптимального управления, описываемую параболической системой.

Рассмотрим задачу $\left(P^{\prime}\right)$ минимизации функционала

$$
I(x, u)=\min _{1 \leqslant i \leqslant n} \int_{T} \int_{\Omega} g_{i}(t, z, x(t, z)) d z d t+\int_{T} \int_{\Omega} f(t, z, u(t, z)) d z d t
$$

на решениях системы

$$
\begin{gathered}
\frac{\partial x}{\partial t}-\sum_{i, j=1}^{N} \frac{\partial}{\partial z_{i}}\left(a_{i j}(t, z) \frac{\partial x}{\partial z_{j}}\right)+a(t, z) x=u, \\
\left.x\right|_{T \times \partial \Omega}=0, \quad x(0, z)=x_{0}(z)
\end{gathered}
$$

с ограничениями

$$
\begin{gathered}
u(t, z) \in U(t, z), \\
\min _{1 \leqslant j \leqslant k} \int_{T} \int_{\Omega} \varphi_{j}(t, z, x(t, z)) d z d t \leqslant b, \\
\min _{1 \leqslant s \leqslant l}\left(\int_{T} \int_{\Omega}\left\langle h_{s}(t, z), u(t, z)\right\rangle_{\mathbb{R}} d z d t+r_{s}\right) \leqslant d .
\end{gathered}
$$

Введем следующие предположения, которые всюду в дальнейшем считаем выполненными.

ГиПотеЗА Н(a). $a_{i j}(t, z), a(t, z) \in L^{\infty}(T \times \Omega), i, j=1, \ldots, N$, и для некоторого $0<\alpha \leqslant c$ и всех $(t, z) \in T \times \Omega, \xi=\left(\xi_{1}, \ldots, \xi_{N}\right) \in \mathbb{R}^{N}$ справедливы неравенства

$$
\begin{gathered}
\sum_{i, j=1}^{N} a_{i j}(t, z) \xi_{i} \xi_{j} \geqslant \alpha \sum_{i=1}^{N} \xi_{i}^{2}, \\
\left|a_{i j}(t, z)\right| \leqslant c, \quad \alpha \leqslant a(t, z) \leqslant c .
\end{gathered}
$$


ГипотезА $\mathrm{H}(\mathrm{g})$. Функции $g_{i}: T \times \Omega \times \mathbb{R} \rightarrow \overline{\mathbb{R}}, i=1, \ldots, h$, являются нормальными интегрантами такими, что:

1) функции $x \rightarrow g_{i}(t, z, x)$ являются вогнутыми при почти всех $(t, z)$;

2) $g_{i}(t, z, x) \geqslant-\beta_{1}\|x\|^{2}-\beta_{2}(t, z)$ п.в., $i=1, \ldots, n, \beta_{1}>0, \beta_{2}(\cdot) \in$ $L^{1}\left(T \times \Omega, \mathbb{R}^{+}\right)$.

ГипотезА $\mathrm{H}(\varphi)$. Функции $\varphi_{j}: T \times \Omega \times \mathbb{R} \rightarrow \overline{\mathbb{R}}, j=1, \ldots, k$, являются нормальными интегрантами такими, что:

1) функции $x \rightarrow \varphi_{j}(t, z, x)$ являются вогнутьми при почти всех $(t, z)$;

2) $\varphi_{j}(t, z, x) \geqslant-\gamma_{1}\|x\|^{2}-\gamma_{2}(t, z)$ п.в., $\gamma_{1}>0, \gamma_{2}(\cdot) \in L^{1}\left(T \times \Omega, \mathbb{R}^{+}\right)$.

ГиПотЕЗА $\mathrm{H}(\mathrm{h})$. Функции $h_{s}(t, z), s=1, \ldots, l$, являются элементами пространства $L^{2}(T \times \Omega)$.

ГиПотеЗА $\mathrm{H}_{0} . x_{0} \in L^{2}(\Omega)$.

Сведем задачу $\left(P^{\prime}\right)$ к задаче $(P)$ в соответствуюших функциональных пространствах.

Пусть $H_{0}^{1}(\Omega)$ - соболевское пространство. Тогда его сопряженным является пространство $H^{-1}(\Omega)$. Положим $V=H_{0}^{1}(\Omega), V^{*}=H^{-1}(\Omega)$. Из теорем вложения для соболевских пространств хорошо известно, что вложения $V \hookrightarrow H \hookrightarrow V^{*}$ непрерывны, плотны и компактны.

Рассмотрим форму Дирихле $a: T \times V \times V \rightarrow \mathbb{R}$, определенную по формуле

$$
a(t, x, y)=\int_{\Omega}\left(\sum_{i, j=1}^{N} a_{i j}(t, z) \frac{\partial x}{\partial z_{i}} \frac{\partial y}{\partial z_{j}}+a(t, z) x y\right) d z .
$$

Из неравенств (5.5), (5.6) следует, что

$$
\begin{gathered}
|a(t, x, y)| \leqslant c_{2}\|x\|_{V}\|y\|_{V}, \quad t \in T, \\
c_{1}\|x\|_{V}^{2} \leqslant c_{1} a(t, x, y)
\end{gathered}
$$

при некоторых $c_{1}>0, c_{2}>0$ (см., например, [3], [20]). Воспользовавшись (5.7)-(5.9), получим, что сушествует оператор $A(t): V \rightarrow V^{*}$, определенньй по правилу

$$
\langle y, A(t) x\rangle=a(t, x, y),
$$

$A(t) \in \mathscr{L}\left(V, V^{*}\right), t \in T$, для которого имеют место все гипотезы $\mathrm{H}(\mathrm{A})$.

Как обычно, под решением уравнения (5.1) при фиксированном $u \in L^{2}(T \times \Omega)=$ $L^{2}\left(T, L^{2}(\Omega)\right)$ мы понимаем обобшенное решение, т.е. решение уравнения $(0.1) \mathrm{c}$ оператором $A(t)$, определенным по формуле (5.10) (см., например, [3], [20]).

Решением управляемой системь (5.1), (5.2) является пара $(x, u), u \in$ $L^{2}(T \times \Omega)$, такая, что $x$ является решением уравнения $(0.1)$ и почти всюду на $T \times \Omega$ имеет место включение (5.2).

Пару $\left(x^{*}, u^{*}\right), x^{*} \in C\left(T, L^{2}(\Omega)\right), u^{*} \in L^{2}(T \times \Omega)$, назовем допустимой для задачи $\left(P^{\prime}\right)$, если она является решением управляемой системы $(5.1),(5.2)$ и удовлетворяет ограничениям (5.3), (5.4).

Сделаем еще одно предположение. 
ГипотеЗА Н(I). Существует допустимая пара $\left(x^{*}, u^{*}\right)$ задачи $\left(P^{\prime}\right)$ такая, что

$$
I\left(x^{*}, u^{*}\right)<\infty \text {. }
$$

Всюду в дальнейшем считаем, что гипотезы $\mathrm{H}(\mathrm{a}), \mathrm{H}(\mathrm{g}), \mathrm{H}(\mathrm{f}), \mathrm{H}(\mathrm{U}), \mathrm{H}(\varphi), \mathrm{H}(\mathrm{h})$, $\mathrm{H}_{0}$ и $\mathrm{H}(\mathrm{I})$ имеют место.

Теорема 5.1. Если $\alpha_{1}>2 \beta_{1}$, то задача $\left(P^{\prime}\right)$ имеет решение.

ДокАЗАТЕЛЬСтво. Обозначим

$$
\begin{aligned}
& G(x)=\min _{1 \leqslant i \leqslant n} \int_{T} \int_{\Omega} g_{i}(t, z, x(t, z)) d z d t \\
& \Phi(x)=\min _{1 \leqslant j \leqslant k} \int_{T} \int_{\Omega} \varphi_{i}(t, z, x(t, z)) d z d t \\
& Q(u)=\min _{1 \leqslant s \leqslant l}\left(\int_{T} \int_{\Omega}\left\langle h_{s}(t, z), u(t, z)\right\rangle_{\mathbb{R}} d z d t+r_{s}\right) .
\end{aligned}
$$

Теорема будет доказана, если мы покажем, что функции $G(x), \Phi(x), Q(u)$ удовлетворяют гипотезам $\mathrm{H}(\mathrm{G}), \mathrm{H}(\Phi), \mathrm{H}(\mathrm{Q})$.

Пусть $x \in C\left(T, L^{2}(\Omega)\right)$. Так как функция $(t, z) \rightarrow x(t)(z)=x(t, z)$ является измеримой, а функции $(t, z, x) \rightarrow g_{i}(t, z, x)$ являются нормальньми интегрантами, то функции $(t, z) \rightarrow g_{i}(t, z, x(t, z))$ измеримы. Воспользовавшись гипотезой $\mathrm{H}(\mathrm{g})$, получим, что функция $G: C\left(T, L^{2}(\Omega)\right) \rightarrow \overline{\mathbb{R}}$ определена, является вогнутой и

$$
\begin{gathered}
-\infty<G(x) \leqslant+\infty, \\
G(x) \geqslant-\beta_{1}\|x\|_{C\left(T, L^{2}(\Omega)\right)}^{2}-\beta_{2},
\end{gathered}
$$

где $\beta_{2}=\int_{T} \int_{\Omega} \beta_{2}(t, z) d z d t$.

Из гипотезы Н(I), (1.3), (5.11) следует, что

$$
G\left(x^{*}\right)<\infty
$$

Воспользовавшись (5.12), (5.13) и предложением 2.5 из [13; гл. 1, §2] (применительно к функции $-G(x))$, мы получаем, что $G(x)$ является вогнутой непрерывной функцией. Тем самым, для функции $G(x)$ выполняется гипотеза $\mathrm{H}(\mathrm{G})$.

Аналогично можно показать, что $\Phi(x)$ является вогнутой непрерывной функцией из $C\left(T, L^{2}(\Omega)\right)$ в $\mathbb{R}$. Следовательно, гипотеза $\mathrm{H}(\Phi)$ имеет место для функции $\Phi(x)$.

И наконец, так как функции $\int_{T} \int_{\Omega}\left\langle h_{s}(t, z), u(t, z)\right\rangle_{\mathbb{R}} d z d t, s=1, \ldots, l$, являются непрерывными линейными функционалами на $L^{2}(T \times \Omega)$, то функция $u \rightarrow Q(u)$ является вогнутой непрерывной функцией из $w-L^{2}(T \times \Omega)=w-L^{2}\left(T, L^{2}(\Omega)\right)$ в $\mathbb{R}$. Тем самым, гипотеза $\mathrm{H}(\mathrm{Q})$ имеет место для функции $Q(u)$. Теперь теорема 5.1 вытекает из основной теоремы. Теорема доказана.

В заключение отметим, что подобная задача без ограничений $(0.4),(0.5)$ и при более жестких предположениях рассматривалась в [9]. 


\section{Список литературы}

1. Янг Л. Лекции по вариационному исчислению и теории оптимального управления. М.: Мир, 1979.

2. Габасов P., Кириллова Ф.M. Методы оптимального управления // Итоги науки и техн. Совр. пробл. матем. Т. 6. М.: ВИНИТИ, 1976. С. 133-261.

3. Лионс Ж.-Л. Оптимальное управление системами, описываемыми уравнениями в частных производных. М.: Мир, 1972.

4. Ahmed N. U., Teo K.L. Optimal control of distributed parameter systems. New York: North-Holland, 1981.

5. Литвинов В.Г. Оптимизация в эллиптических граничных задачах с приложениями к механике. М.: Наука, 1987.

6. Иваненко В. И., Мельник В. С. Вариационныеметоды в задачах управления для систем с распределенньми параметрами. Киев: Наукова думка, 1988.

7. Фурсиков $A$. B. Оптимальное управление распределенньми системами. Теория и приложения. Новосибирск: Научная книга, 1999.

8. Suryanarayn M.B. Existence theorems for optimization problems concerning linear, hyperbolic partial differential equations without convexity conditions // J. Optim. Theory Appl. 1976. V. 19. № 1. P. 47-61.

9. Raymond J.P. Existence theorems without convexity assumptions for optimal control problems governed by parabolic and elliptic systems // Appl. Math. Optim. 1992. V. 26. P. 39-62.

10. Bressan A., Flores F. Multivariable Aumann integrals and controlled wave equation // J. Math. Anal. Appl. 1995. V. 189. № 2. P. 315-334.

11. Толстоногов Д. А. О минимуме в вариационных эллиптических задачах без предположения вьпуклости // Матем. заметки. 1999. Т. 65. № 1. С. 130-142.

12. Толстоногов A. A. Теорема существования оптимального управления в задаче Гурса-Дарбу без предположения выпуклости // Изв. РАН. Сер. матем. 2000. Т. 64. № 4. C. $163-182$.

13. Экланд И., Темам Р. Выпуклый анализ и вариационные проблемы. М.: Мир, 1979.

14. Zeidler E. Nonlinear functional analysis and its applications. II. New York: Springer-Verlag, 1990.

15. Бурбаки Н. Топологические векторные пространства. М.: ИЛ, 1959.

16. Brezis H. Opérateurs maximaux monotones et semi-groupes de contractious dans les espaces de Hilbert. Amsterdam: North-Holand, 1973.

17. Толстоногов A. A. К теореме Скорца-Драгони для многозначных отображений с переменной областью определения // Матем. заметки. 1990. Т. 48. № 5. С. 109-120.

18. Himmelberg C. J. Measurable relation // Fundamenta Math. 1975. V. 87. P. 53-72.

19. Иоффе А.Д., Тихомиров В. М. Теория экстремальных задач. М.: Наука, 1974.

20. Cesari L., Hou S. H. Existence of solution and existence of optimal solutions. The quasilinear case // Rend. Circ. Math. Palermo. (2). 1985. V. 34. № 1. P. 5-45.

Институт динамики систем и теории управления СО Р AH, 은 г. Иркутск

27.09 .2000

E-mail: aatol@icc.ru 